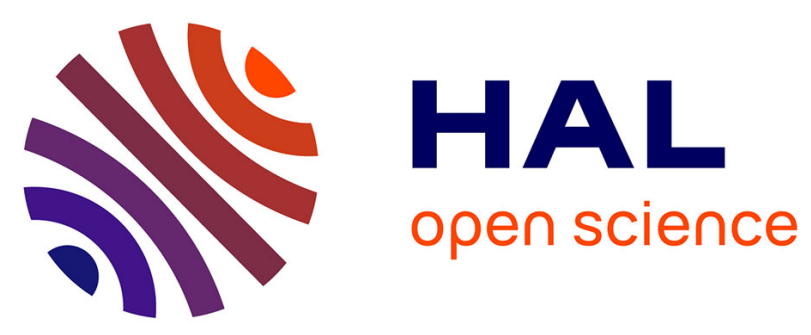

\title{
Externaliser la contrainte
}

Aurélie Tricoire

\section{To cite this version:}

Aurélie Tricoire. Externaliser la contrainte: Le dispositif de pilotage d'un projet de recherche communautaire. Terrains et Travaux: Revue de Sciences Sociales, 2006, 11, pp.122-139. hal-00186673

\section{HAL Id: hal-00186673 https://hal.science/hal-00186673}

Submitted on 11 Nov 2007

HAL is a multi-disciplinary open access archive for the deposit and dissemination of scientific research documents, whether they are published or not. The documents may come from teaching and research institutions in France or abroad, or from public or private research centers.
L'archive ouverte pluridisciplinaire HAL, est destinée au dépôt et à la diffusion de documents scientifiques de niveau recherche, publiés ou non, émanant des établissements d'enseignement et de recherche français ou étrangers, des laboratoires publics ou privés. 
Externaliser la contrainte Le dispositif de pilotage d'un projet de recherche communautaire (enquête)

\section{Introduction}

L'objet auquel nous allons nous intéresser est un dispositif d'action publique qui sert à piloter les projets $\mathrm{du} 6^{\text {ème }}$ programme-cadre de recherche et développement technologique (PCRDT). Ce dispositif est «à la fois technique et social » puisqu'il est agencé autour d'un contrat collectivement élaboré et négocié et qu'il "organise les rapports sociaux spécifiques » qui émergent entre la Délégation Générale Recherche (DG Recherche), responsable de la mise en œuvre du PCRDT, et un consortium de recherche, porteur de projet (Lascoumes et Le Galès, 2004, p.13). Les acteurs (les co-contractants) et actants (les formes contractuelles mobilisées) constituent un collectif sociotechnique qui s'articule autour du projet de recherche (Latour et Woolgar, 1988). C'est sur ce collectif sociotechnique que porte notre analyse.

La question qui nous intéresse est de savoir ce que le fonctionnement de ce collectif et le mode de pilotage que l'existence de dispositifs sociotechniques induit, impliquent en termes d'organisation, d'engagement et de justification des pratiques scientifiques et politiques. Dans une première partie, nous montrons que l'externalisation du pilotage $\mathrm{du}$ projet, initiée par l'administration communautaire, s'appuie sur un dispositif sociotechnique contractuel spécifique. Dans une seconde partie, nous étudions comment ce dispositif, complété et étendu, contribue à l'organisation interne du consortium en fournissant un cadre pour l'action collective de recherche tout en ménageant des espaces de libertés, propres à assurer un pilotage pragmatique. Cela pose plus généralement la question de l'efficacité de l'externalisation du pilotage des projets et du rôle que joue le dispositif contractuel autour duquel s'organise ce transfert de compétences de l'administration vers les consortia à la fois en termes de gouvernement de la recherche communautaire et de management des activités scientifiques.

Notre réflexion s'appuie sur l'étude du projet "EA-BIOFILMS», qui s'inscrit dans la sousthématique NEST (New and Emerging Sciences and Technologies) du $6{ }^{\text {ème }}$ PCRDT et qui rassemble sept groupes de chercheurs basés en France, Allemagne, Belgique, Italie et au Portugal. Ces équipes de chercheurs étudient de fines couches de micro-organismes, ou biofilms, qui se déposent sur n'importe quel milieu non stérile. Le projet s'intéresse aux biofilms qui présentent la propriété chimique d'augmenter sensiblement l'intensité d'un courant électrique. L'objectif est de trouver des milieux propices au développement de biofilms électrochimiquement actifs puis d'identifier les micro-organismes qui les composent afin de parvenir à les cultiver en laboratoire.

Notre travail empirique est basé sur quatre sources de données ${ }^{1}$. Nous avons consulté les archives $\mathrm{du}$ projet qui renseignent les phases d'élaboration et de finalisation du contrat. Nous avons également réalisé une vingtaine d'entretiens avec les personnes impliquées dans le projet, aussi bien au niveau de l'administration communautaire que du consortium de recherche. Nous avons par ailleurs recueilli l'ensemble des courriers électroniques échangés entre les chercheurs et avec l'administration communautaire depuis la phase de soumission du projet en avril 2003 jusqu'à aujourd'hui ${ }^{2}$. Enfin, nous avons assisté aux réunions de pilotage organisées entre avril 2004 et avril 2006, et avons pu accéder à l'ensemble des « Minutes » des réunions qui se sont déroulées depuis le lancement du projet en septembre 2004. La diversité des informations recueillies par ces canaux

1 Le travail de collecte des données a été largement facilité par la signature en avril 2005 d'un accord de confidentialité avec le consortium de recherche. Cet accord nous assure un libre accès aux réunions et aux documents relatifs au projet, sous réserve de respecter la confidentialité des résultats de recherche du projet. Ce texte a lui-même été soumis au comité de pilotage du projet afin de vérifier qu'aucune information brevetable ou publiable ne serait divulguée par ce biais.

2 Le travail de terrain entrepris est en cours, le projet EA-Biofilms ne devant se terminer qu'en septembre 2007. 
nous a permis d'obtenir une vision polycentrée du projet qui rend plus aisée la compréhension du rôle du dispositif sociotechnique mobilisé.

\section{Externaliser le pilotage}

\section{Garantir l'implémentation du projet}

Selon l'article 1101 du Code civil belge ${ }^{3}$, «le contrat est une convention par laquelle une ou plusieurs personnes s'obligent envers une ou plusieurs autres à donner, à faire ou ne pas faire quelque chose ». Ainsi, la raison d'être du Contrat $N^{\circ} 508866$ (NEST) consiste à fixer par écrit un engagement mutuel :

The European Community, and the contractor acting as coordinator of the consortium [...] and the other contractors identified in Article 1.2 below, HAVE AGREED to the following terms and conditions established in this contract and its annexes (the "contract").

\section{Préambule du Contrat}

Cet accord des parties concerne des dispositions volontairement prises afin de réaliser le projet décrit dans le contrat. La rédaction de ce document, lors de la phase finale de sélection du projet, a offert aux chercheurs et à leur futur partenaire financier une opportunité d'échanger leurs points de vue. En effet, l'Annexe I, partie du contrat qui décrit avec le plus de précisions le projet EABiofilms, résulte d'un important travail collectif de transformation qui a été réalisé au cours des différentes phases de sélection du projet. La proposition initiale, courte, qui avait attiré l'intérêt des évaluateurs de la DG Recherche, a été étoffée pour aboutir à une proposition complète, qui a su convaincre de la qualité du projet dans la deuxième phase de sélection. C'est ce document qui a été repris à partir de décembre 2003 comme base de discussion entre le consortium et l'administration afin de finaliser l'Annexe I du contrat. Celui-ci a finalement été signé en juin 2004 et le projet lancé au $1^{\text {er }}$ septembre de la même année, pour une durée de trois ans. Résultat d'un travail collectif, ce dispositif juridique peut donc être qualifié d'objet-frontière (Leigh Star et Griesemer, 1989). En effet, son élaboration a été adossée à l'obtention d'un consensus relatif au contenu technique, scientifique et administratif du projet. Le dispositif sociotechnique que constitue le contrat se distingue par son caractère contraignant, lié à sa nature contractuelle, et c'est justement l'influence de cette contrainte sur le pilotage du projet que nous allons étudier.

Le contrat EA-Biofilms per se est un document standard ${ }^{4}$ de quatorze articles : huit sont à compléter tandis que les six autres ne varient pas d'un projet à l'autre. Cinq annexes complètent le corps du contrat en en spécifiant le contenu. Seule l'Annexe I n'est pas standardisée. Elle constitue le seul élément de différenciation des projets puisqu'elle décrit le travail de recherche à faire ainsi que les conditions spécifiques de management (Annexe I.6 Project management and exploitation/dissemination plans), de reporting ${ }^{5}$ (Annexe I.7.5 Deliverables ${ }^{6}$ list) et de gestion financière (Annexe I.8 Project resources and budget overview) du projet. La structure de ce document est pré-établie par l'administration. Le découpage des activités de recherche en tâches, elles-mêmes regroupées en work packages ${ }^{7}$, l'évaluation de la durée et de la charge de travail en personne par mois ainsi que la répartition des moyens budgétaires entre les partenaires font partie des passages obligés communs à tous les projets de recherche communautaires. Le temps et les moyens de la recherche sont ainsi planifiés dans le contrat.

\footnotetext{
Le contrat établi entre l'administration communautaire et le consortium de recherche est soumis à la loi belge (Article 12 du Contrat).

La version standard utilisée pour tous les contrats du 6ème PCRDT est téléchargeable sur : http://www.cordis.lu/fp6/find-doc-specific.htm\#modelcontracts

Il s'agit de l'activité de production et de soumission de rapports en vue de leur évaluation.

Ce terme désigne l'ensemble des documents (Minutes de réunion, rapports périodiques, rapports finaux, rapports d'activité, prototypes, etc.) que le consortium doit « délivrer » c'est-à-dire fournir à la Commission.

Ce sont des groupes de tâches planifiées.
} 
L'administration communautaire n'accorde son soutien financier qu'à des projets qui se conforment au cadre contractuel qu'elle a façonné. Cette standardisation formelle des projets permet d'une part de les rendre commensurables (Paradeise, 1998) afin de pouvoir les sélectionner et d'autre part d'informer les chercheurs sur les éléments qui doivent figurer dans une proposition de projet :

We could say: "ok follow any structure and we will see anyway what is going on". But if you are dealing with a lot of projects I think that it is easier for us. And it is also easier for them [the proposers] too, to a certain degree, to follow the structure [of Annex I] because otherwise you never now if it is sufficient or not. [...] So I think if there is a clear structure it is much easier for them.

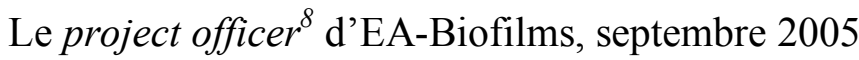

Cette standardisation contribue à la construction d'un cadre cognitif (Callon, 1999, p.407) commun, qui permet de définir ce qu'un projet de recherche communautaire doit être. Ce cadre cognitif permet aux services de la Commission et aux proposants ${ }^{9}$ de réaliser un gain de temps en facilitant l'émergence d'un accord quant aux objectifs à atteindre ${ }^{10}$. Mais ce document ne peut pas prévoir toutes les évolutions possibles du projet (Dupuy, Eymard-Duvernay, Favereau, Orléan, Salais et Thévenot, 1989). La Commission ménage donc une marge de manoeuvre au consortium afin de lui assurer les moyens de mener le projet à bien. L'administration communautaire fait donc confiance aux compétences des chercheurs pour piloter le projet. Dans le cas du projet EA-Biofilms, cela a débouché sur la mise en place par le consortium d'une stratégie de prise en compte et d'évaluation du risque :

The management strategy is based on two key words: flexibility and risk assessment [...]. The partners [...] build a project that will carefully analyse the results through several milestones and offer contingency / back-up solutions to continue the project.

Annexe I.7 Workplan / Management activity du Contrat

Quatre risques principaux, non prévus dans le contrat, sont explicitement placés sous la responsabilité du consortium. $1^{\circ}$ ) Le consortium doit se fixer des règles relatives à l'adhésion, à $1^{\prime}$ 'exclusion et au fonctionnement du consortium. $2^{\circ}$ ) Il est chargé de gérer les questions de confidentialité, de diffusion des résultats et de propriété intellectuelle. $3^{\circ}$ ) Le consortium est responsable du lancement d'une procédure de certification des dépenses qui servira à faire valider le bilan financier du projet. $4^{\circ}$ ) La cohérence du contenu scientifique du projet par rapport aux objectifs initiaux et le management des activités de recherche relèvent de la responsabilité des chercheurs.

On peut se demander pourquoi l'administration communautaire, après avoir fixé un cadre contractuel relativement strict, choisit d'externaliser aux consortia la gestion de ces questions, pourtant centrales. En effet, des accords issus de consensus négociés entre les deux parties pourraient être transformés en décisions formelles par l'autorité du project officer plutôt que par celle du consortium. À cela, nous avons trouvé deux explications principales. D'une part, le caractère imprévisible de l'activité de recherche impose des modes de gestion relativement souples. D'autre part, la gestion des projets de recherche doit être adaptée aux contraintes communautaires, notamment en termes de ressources humaines. Ce problème a été pointé lors de l'élaboration du $6^{\text {ème }}$ PCRDT :

Dans le précédent programme-cadre, la Commission a géré quelque chose comme quarante mille projets... il n'y a pas beaucoup de fonctionnaires [européens] [...]

Également appelé scientific officer, il s'agit du fonctionnaire européen en charge du projet au sein de la DG Recherche. Il a été l'interlocuteur privilégié du consortium lors des phases de sélection du projet. Il est assisté d'un financial officer, qui s'occupe de la partie comptable du projet.

9 Il s'agit d'un néologisme, adapté du terme anglais proposer, utilisé par l'administration communautaire pour désigner les personnes lui ayant soumis une proposition de projet.

10 Les objectifs sont fixés en termes d'engagement de moyens et non de résultats, la recherche scientifique étant une activité par nature imprévisible (Foray, 2000). 
[L']idée des nouveaux instruments c'est de réduire le nombre de projets de quarante milles à [...] quatre milles. Et aussi de donner plus de liberté au consortium pour la gestion.

Le Conseiller scientifique de la Représentation Permanente espagnole auprès de l'Union Européenne $^{11}$, avril 2003

Le dispositif contractuel a donc été mis en place afin d'alléger la charge de travail de la DG Recherche en faisant prévaloir de nouveaux critères de sélection des projets, plus formalistes que substantialistes : la taille et le respect des règles administratives semblent primer sur la qualité scientifique d'un projet.

Toujours dans la perspective de réduire les tâches assurées par l'administration, une seconde modification a été apportée depuis 2002 : les nouveaux contrats rendent désormais obligatoire la validation par un cabinet d'audit indépendant, et ce pour chacun des membres du consortium, des dépenses mentionnées dans le rapport périodique financier (Article $8.2 \mathrm{du}$ Contrat). Auparavant, seule l'administration communautaire prenait l'initiative de procéder au lancement de ce type de contrôle et uniquement lorsqu'elle le jugeait nécessaire. Nous assistons à l'externalisation du travail d'audit financier, ce qui a pour contrepartie d'en permettre la systématisation.

Pourtant, il semble que l'augmentation de la taille des projets et la mise en sous-traitance de la certification financière ne soient pas entièrement parvenues à régler le problème de déficit en ressources humaines diagnostiqué au niveau communautaire :

A project officer is dealing with twenty to... I don't know, there is no upper limit... to fifty projects. [...] So it is very difficult to follow the scientific developments in a very detailed way.

Le project officer d'EA-Biofilms, septembre 2005

Ne pouvant être présente tout au long du déroulement du projet, l'administration a ainsi choisi de forger un dispositif basé sur un cadre fixe et d'en confier le pilotage au consortium de recherche. La Commission délègue ainsi la fonction de garant $\mathrm{du}$ 《 bon ${ }^{12}$ déroulement du projet au dispositif sociotechnique contractuel qu'elle a mis en place à cet effet: le contrat doit permettre aux chercheurs de se rappeler tout au long du projet ce qu'ils se sont engagés à faire. Ce dispositif sociotechnique se substitue à un acteur humain, qui aurait sinon été chargé de cette fonction de rappel à l'ordre.

Mais le choix de la Commission d'externaliser le pilotage du projet au consortium ne peut pas seulement être perçu comme une option adoptée par défaut. Nous verrons dans notre seconde partie l'avantage que ce dispositif de pilotage produit en termes d'intégration de la contrainte de suivi du projet par les chercheurs.

\section{Délégation et réversibilité}

L'administration n'externalise pas le pilotage du projet au consortium en se reposant sur le seul contrat pour garantir le bon déroulement du projet. Elle met également en place un mécanisme de contrôle dont les modalités de fonctionnement sont fixées dans le contrat et dont l'implémentation est sensiblement influencée par des aspects plus relationnels :

Au-delà des procédures contractuelles mise en place par la CE [Communauté Européenne] pour gérer les projets, les personnalités du scientific officer et du coordinateur sont primordiales pour réussir un projet. [...] Il y a donc un aspect humain,

11 La Représentation Permanente de l'Espagne auprès de l'Union Européenne a assuré la Présidence de l'Union du 1er janvier au 1er juillet 2002, lors des négociations du $6^{\text {ème }}$ PCRDT.

12 L'adjectif « bon » renvoie ici à un respect des règles et des objectifs prévus dans le contrat. 
relationnel, d'honnêteté et de confiance réciproque très important entre le représentant de la $\mathrm{CE}$ et le consortium.

Julio Vallès ${ }^{13}$, courrier électronique, mars 2006

Cependant, en cas de difficultés, le project officer n'hésite pas à intervenir et à reprendre sa place dans le pilotage du projet :

My feedback to the consortium is rather limited if there are no problems with the deliverables. If there is any major problem I come back to them.

Le project officer d'EA-Biofilms, courrier électronique, mars 2006

La délégation du rôle de garant de l'implémentation du projet au dispositif contractuel n'est donc pas un processus irréversible, bien au contraire (Latour, 1993a p.65). Pour pouvoir « revenir vers le consortium ", et reprendre la main sur le pilotage, l'administration s'engage à en évaluer régulièrement l'évolution (Article 3.3 de l'Annexe II du Contrat) et exige pour cela que le consortium respecte ses obligations de reporting. En effet c'est sur la base de l'analyse des deliverables, pondérée selon la qualité des relations entretenues entre le consortium et le project officer, que ce dernier décidera du degré de suivi du projet :

Si le scientific officer a des doutes sur la qualité du travail qui est fait, il mandatera un scientific expert pour auditer les deliverables, car lui n'a en règle générale pas les compétences pour juger de la qualité du contenu scientifique du projet.

Julio Vallès, courrier électronique, mars 2006

L'activité de reporting consiste pour le consortium EA-Biofilms à rendre trente deliverables sur la durée totale du projet parmi lesquels trois rapports périodiques (d'activité, de management et financier) pour chacune des deux périodes de reporting définies à l'Annexe II.7.2 du contrat. Ce dispositif non-humain d'inscription (Latour, 1993b) garantit à la Commission qu'elle pourra évaluer le projet en comparant les inscriptions des deliverables avec celles du contrat. Dans le cas de projets ne posant pas de problème quant à la qualité du management, le project officer opère une sélection parmi les deliverables dont il va tenir compte dans le suivi du projet :

In practice I do not have time to read all the deliverables when they are sent to me because they are due. I am in charge of thirty projects so this would be impossible.

Le project officer d'EA-Biofilms, courrier électronique, mars 2006

Dans le cas de EA-Biofilms, perçu comme un projet ne posant «pas de problème » (de l'avis du project officer et du financial officer), seuls les rapports périodiques seront évalués. Il ne devrait donc y avoir que deux évaluations, l'une à mi-parcours et l'autre à la fin du projet. Le project officer subordonne à cette évaluation scrupuleuse la poursuite du projet :

In terms of the project periods, however, progress reports (technical and financial) have to be delivered. These reports should describe or enclose the deliverables (scientific and management) that were covered by the respective project period and here I have to monitor them carefully because at this stage the achievements and results and the deliverables are linked to the acknowledgement of costs and to further payments.

Le project officer d'EA-Biofilms, courrier électronique, mars 2006

Le mécanisme de contrôle du bon déroulement du projet repose sur une évaluation de la qualité du management scientifique et non sur les résultats du projet. Le project officer examine ainsi $a$ posteriori la transcription de tâches réalisées en amont (chronologiquement) et en externe (géographiquement) et il les compare avec celles inscrites dans le contrat :

13 Julio Vallès appartient au cabinet de conseil Impulsion, sous-contractant du consortium EA-Biofilms (Appendix A.2. de l'Annexe I du Contrat). Il est chargé de la gestion administrative du projet. 
À l'issue de [l']analyse [des rapports périodiques], [le projet officer] nous dira s'il accepte les rapports ou non. "Accepter" signifie reconnaître que le programme de travail prévu dans l'annexe technique [Annexe I] a été mis en oeuvre par les partenaires. S'il y avait eu des modifications (sujettes ou non à des amendements du contrat) cela signifie aussi que les modifications sont acceptées.

Julio Vallès, courrier électronique, mars 2006

Une fois la partie managériale positivement évaluée, c'est-à-dire lorsque le project officer considère que les tâches qui ont été entreprises sont satisfaisantes par rapport à ce qui était prévu et, le cas échéant, lorsque les décisions prises hors du cadre du contrat sont jugées pertinentes par rapport aux objectifs de celui-ci, la partie financière est soumise à l'appréciation de l'administration. En cas d'évaluation négative des rapports, le projet peut au contraire être menacé :

The Commission may terminate the contract or the participation of a contractor $[\ldots] \mathrm{b}$ ) where in accordance with the provisions of Article II.8, the required reports are not approved by the Commission.

\section{Article II.15.5 de l'Annexe II du Contrat}

L'intervention humaine se borne ici à un contrôle d'adéquation entre deux dispositifs, les deliverables, artefact d'une réalité passée et relatée, et le contrat, artefact d'une réalité fictive et anticipée en amont du lancement du projet. Ces artefacts sociotechniques jouent ici le rôle de médiateur entre le consortium et le project officer (Latour, 1993a). Ils permettent à l'administration de participer au pilotage du projet de manière discrète, à travers des acteurs ponctuellement impliqués, mais paradoxalement continue, via le dispositif contractuel qui garantit, par sa nature contraignante, le bon déroulement du projet.

C'est le caractère réversible et ajustable de ce dispositif de pilotage, relativement classique puisque basé sur une externalisation contractuellement cadrée, qui en constitue la caractéristique principale. Le fait que l'administration puisse intervenir à tout moment dans le pilotage du projet induit une contrainte plus forte pour le consortium que si l'administration occupait la position fixe d'un acteur continûment impliqué. Il s'agit donc pour les chercheurs de fournir des preuves convaincantes de leur capacité à piloter afin de conserver le degré de liberté que la situation créée par l'externalisation leur offre. Mener de front une bonne gestion administrative et managériale du projet et les activités scientifiques devient ainsi un réel enjeu pour les membres du consortium. La manière dont le consortium intègre et gère cette contrainte sera l'objet de notre seconde partie.

\section{Intégrer et gérer la contrainte}

\section{Entre pragmatisme et respect des engagements}

Si l'administration communautaire n'intervient que ponctuellement, ici lors des périodes de reporting, dans le pilotage, elle encourage fortement les porteurs de projet à se constituer en collectivité (Paradeise, 1998), en se dotant d'un accord interne, le consortium agreement :

The consortium shall make appropriate arrangements for its internal operation and management, which may include any intellectual property provisions. To this end, a consortium agreement may be established, which will cover any other additional aspects necessary for the consortium management and the implementation of the project. 
L'administration a ainsi rédigé et diffusé un document intitulé "Sixth Framework Programme. Checklist for a Consortium Agreement ${ }^{14}$ qui, en quatorze pages, se propose d'aider les consortia qui souhaiteraient se lier par un consortium agreement :

This checklist is provided to assist contractors in an EC-funded project to identify issues that may arise during the implementation of a research project and which may be facilitated or governed by means of the Consortium Agreement. Introduction, "Sixth Framework Programme. Checklist for a Consortium Agreement »

Il s'agit d'encourager et de soutenir, par actants interposés, la prise en charge par le consortium des tâches de pilotage externalisées par la Commission. Le consortium agreement traite précisément des risques que le contrat plaçait sous la responsabilité du consortium. L'administration communautaire étant consciente de l'incomplétude du dispositif contractue ${ }^{15}$ auquel elle a délégué l'implémentation du projet, elle propose aux chercheurs, à travers la mise à disposition de cette check-list, un appui, véritable dispositif cognitif d'aide (Norman, 1992). La check-list vient ici faciliter l'élaboration du consortium agreement, document complémentaire au contrat. Ce document doit permettre de combler les vides laissés dans le contrat afin que le consortium puisse se reposer sur des cadres aussi complets que possible pour guider son action pendant le projet.

Le projet EA-Biofilms a suivi l'incitation de l'administration et a adopté le consortium agreement $N^{\circ} E A$-Biofilms $/ 508866$ lors de la réunion de lancement du projet en septembre 2004. Il s'agit d'un document de quarante-huit pages qui dote le consortium d'une instance de décision, le steering committee, littéralement " comité de pilotage » dont le rôle consiste à prendre en charge les quatre sources de risque que nous avons identifiées plus haut (Article 4.1.6 Role du Consortium Agreement). Le consortium charge le steering committee de prendre les décisions que le contrat ne permet pas de trancher, c'est-à-dire de suivre de manière pragmatique le pilotage du projet. Les deux dispositifs sociotechniques, que sont le contrat et le consortium agreement, constituent donc les supports des décisions rendues par le steering committee. Ainsi, face à un imprévu (les basses températures de l'hiver 2004), le steering committee a dû arbitrer entre deux engagements initiaux (tester un sol ou respecter le calendrier initial du projet). Au final, il a été décidé que le sol serait testé l'année suivante et donc que les expérimentations qui devaient découler de cette tâche seraient reportées. Prendre une décision c'est donc trouver un équilibre entre le respect d'engagements contractuels initiaux et la nécessaire gestion des évènements imprévus afin de mener le projet à bien.

Les membres du steering committee, quotidiennement pris par leurs activités scientifiques, ne se saisissent pas spontanément de questions qui concernent le déroulement global du projet. C'est ici qu'intervient le coordinateur à qui chacun des membres du consortium s'adresse et qui dispose ainsi d'une vision d'ensemble du projet. C'est lui qui saisit le steering committee. Coordonner un projet constitue une tâche extrêmement lourde puisqu'elle consiste à s'assurer que les engagements qui ont été consignés ${ }^{16}$ dans le contrat sont respectés par les participants, c'est-à-dire que chacun mobilise les moyens nécessaires à la réalisation des tâches planifiées (Article 6.1 Co-ordinator de l'Annexe I du Contrat). Cette tâche de coordination est d'autant plus importante que chacun a tendance à travailler selon son idée, ses méthodes et techniques de travail et ses envies, ce qui est susceptible de mettre en danger la cohérence du travail collectif :

I think that they both [the coordinator and his assistant] do a very good job and I know from other projects that we had here before that it is very hard to get so many people together. [...] But I think [the coordinator] in a scientific way can very well arrange that all the other people are working together in the group and that there is exchange and

14 Le document est disponible sur : http://www.europa.eu.int/comm/research/fp6/model-contract/pdf/checklist_en/pdf

15 Les règles et procédures sont « incomplètes par nature» (il est impossible de tout prévoir) et « par construction » (seules les situations routinisées font l'objet de règles) (Paradeise, 1998 p.208).

16 Consigner c'est « signer avec » c'est-à-dire inscrire durablement, ici dans le contrat ; et également « laisser à la consigne » afin de se laisser la possibilité de revenir plus tard sur un sujet, comme le permet le consortium agreement. 
especially Julio Vallès [the coordinator assistant] can manage that they are taking care of the official staff, of the organisation part. And I think it is very important that such people are involved in the project.

Un membre du projet EA-Biofilms, décembre 2005

Cet extrait d'entretien témoigne du caractère peu habituel de la présence d'un assistant-coordinateur travaillant spécifiquement sur les aspects organisationnels et administratifs du projet. L'émergence de cette nouvelle fonction facilite la subdivision des tâches de coordination. Le coordinateur peut se consacrer davantage aux tâches à caractère scientifique tandis que la coordination administrative relève de la responsabilité de son assistant. Le pilotage du projet EA-Biofilms est concrètement assuré par deux personnes et l'une d'elles n'est impliquée dans le projet qu'avec la seule mission d'en assumer la gestion administrative, à l'exclusion de tout autre activité :

Le but c'est quand même que le projet marche, avant tout. Donc mon rôle, c'est de faire respecter les référentiels, c'est-à-dire le contrat avec la Commission, l'annexe technique et l'accord de consortium. [...] Il faut être rigoureux dans la traçabilité des choses, s'assurer que les gens font ce qu'ils ont dit qu'ils feraient donc ce qui était prévu. J'ai un rôle d'arbitre, je suis garant du référentiel. [...]. Je cadre par rapport à ce qui était prévu dans le projet. J'essaye d'éviter les débordements...

Julio Vallès, octobre 2005

Ainsi, nous observons un processus de distribution (Latour, 1993a), par délégations successives, de la fonction de pilotage. D'abord délégué par l'administration communautaire au dispositif contractuel, puis intégré comme base du fonctionnement des instances de décision du consortium, le suivi du pilotage est finalement confié par le consortium, en interne, au coordinateur qui l'a luimême partiellement sous-traité (Appendix A.2 Sub-contracting de l'Annexe I du Contrat). La gestion de la contrainte engendrée par la réversibilité de l'externalisation du pilotage est donc peu à peu intégrée et internalisée par le consortium. Externaliser le pilotage du projet en mobilisant un dispositif contractuel contraint les chercheurs à se saisir des problèmes de pilotage et à les régler en interne, c'est-à-dire à en devenir responsables.

\section{Quand piloter c'est canaliser}

Piloter un projet de recherche consiste à cadrer pour " éviter les débordements » comme le souligne plus haut Julio Vallès. Les cadres du projet, qu'ils soient cognitifs (savoir-faire préexistant, existence d'une communauté scientifique, conception commune du projet de recherche communautaire) ou organisationnels (planification des activités, organisation des équipes et du consortium, évaluation), permettent de gérer les évènements imprévus, comme le gel, en interne (Callon, 1999, p.411). Jusqu'à présent aucun événement n'est parvenu à déborder les cadres du projet. Cependant, les connexions avec le monde extérieur constituent des sources de débordements des cadres, le pire d'entre eux étant la réussite de concurrents. Cette notion de cadragedébordement (Callon, 1999) éclaire le fonctionnement du pilotage d'un projet communautaire de recherche : piloter c'est canaliser dans des cadres fixes et rigides, des évènements imprévisibles et fluctuants.

Pour ce faire, le rappel de la contrainte liée à la réversibilité de l'externalisation du pilotage est distribué entre les acteurs humains et non-humains, qui se complètent et parfois se contredisent. Les prescriptions des acteurs s'ajustent lors des réunions de pilotage. Les « référentiels » (c'est-à-dire le contrat, le consortium agreement et les différents deliverables) sont mobilisés à chaque réunion afin de vérifier l'avancement réel des tâches planifiées. Au lancement du projet, n'ont été mises en œuvre que les tâches prévues dans l'Annexe I. Les six premiers mois passés, les membres du consortium ont rendu compte de leur travail ${ }^{17}$ lors de la première réunion de pilotage de mars 2005.

17 Selon nos observations, il s'agit aussi bien de travail scientifique (expérimentations, recherche bibliographique) que de management du projet (rédaction de deliverables, règlement de questions financières). 
Les membres du steering committee, équipé chacun d'un exemplaire du contrat, qu'ils appellent entre eux la «Bible », ont évalué ces comptes-rendus en fonction de ce qui était initialement prévu. L'achèvement de chaque tâche est validé par un vote et consigné dans les Minutes de la réunion qui sont ensuite envoyées aux membres du consortium ainsi qu'au project officer. Le steering committee se fie pour la première période de six mois uniquement au cadre contractuel.

Dans un second temps, les tâches planifiées dans l'Annexe I pour les six mois suivants ont été examinées pour décider, toujours après un vote du steering committee, si les résultats obtenus jusqu'alors étaient suffisants pour lancer de nouvelles expérimentations. Concrètement, l'assistantcoordinateur reprend chacune des tâches initialement planifiées dans l'Annexe I et le steering sommitte décide pour chacune s'il faut la prolonger, la compléter, et/ou lancer la tâche suivante. Les personnes concernées par ces tâches annotent leur «Bible» et confirment que le temps nouvellement imparti pour réaliser le travail est acceptable, en fonction de leurs autres obligations. Le steering committee confronte cette fois le contrat avec la réalité du déroulement du projet et statue pragmatiquement, comme l'illustre l'exemple du sol gelé qui a conduit au report d'une partie des tâches planifiées à l'année suivante. Le calendrier des tâches est ainsi mis à jour à l'aune des résultats obtenus pendant les périodes précédentes. Le nouveau calendrier est consigné dans les Minutes des réunions et vient grossir la pile des deliverables qui serviront de référentiel pour l'évaluation, lors de la prochaine réunion, des tâches réalisées.

Nous observons donc un travail cyclique d'auto-évaluation, produisant semestriellement de nouveaux cadres pour l'action : en se basant sur ce qui était prévu, le steering committee évalue ce qui a été fait et réoriente les actions à venir à la fois en fonction des engagements initiaux et de ce que les résultats actuels permettent d'entreprendre. Le contrat et le consortium agreement sont donc des dispositifs sociotechniques non-humains en déploiement, puisque évolutifs (annotables et cumulatifs) et amenés de circuler (entre les membres du consortium et jusqu'au project officer) tout au long du déroulement du projet. Le steering committee se charge d'étendre les cadres initiaux en y intégrant les débordements, c'est-à-dire l'évolution naturelle bien qu'imprévisible, du projet. Ce type de pilotage permet de maintenir le projet dans une certaine continuité par rapport aux objectifs initiaux sans pour autant en compromettre le bon déroulement: l'équilibre entre cadres et débordements est ainsi maintenu de manière dynamique.

Les cadres du projet sont donc utilisés comme une check-list évolutive que l'on mobilise après l'action pour vérifier que l'on a bien fait ce qui était prévu (Norman, 1992 " checklist as checks ») ; mais également avant l'action, afin de s'orienter, de déclencher des réponses ou des procédures prescrites spécifiquement pour faire face à une situation donnée (Norman, 1992 «checklist as "triggers" »). Le pilotage du projet s'effectue grâce à un va-et-vient régulier (tous les six mois) entre les check-lists de vérification et de déclenchement opéré par le steering committee et ponctuellement évalué par le project officer. Le consortium a donc parfaitement intégré la contrainte engendrée par le contrat dans son mode de fonctionnement puisqu'il ne cesse de produire des justificatifs de son action afin de montrer à l'administration que les engagements pris dans le contrat sont bien respectés et avant tout afin de mener le projet à terme, objectif premier de leur engagement.

\section{Conclusion}

Nous soulignerons pour conclure les deux principaux résultats de notre enquête. Le premier concerne la manière dont l'administration communautaire administre les projets de recherche qu'elle finance. En effet, depuis le $6^{\text {ème }}$ PCRDT, la Commission a fait le choix de renforcer la tendance à l'externalisation du pilotage des projets vers les consortia de recherche, ce qui en modifie sensiblement le suivi. L'administration a ainsi choisi de déléguer le rôle de suivi du projet à un dispositif sociotechnique contractuel. Sur la base de ce contrat, élaboré collectivement dès la 
phase de sélection du projet, l'administration met en place un mécanisme de contrôle, ponctuel mais efficace, qui lui permet si nécessaire de reprendre la main sur le pilotage du projet. Le degré d'implication de l'administration dans le pilotage du projet est donc ajustable en fonction du déroulement du projet et c'est cette réversibilité de l'externalisation du pilotage qui en fait la spécificité.

Le second résultat de cette recherche concerne la gestion par le consortium de recherche de la contrainte qui résulte de la réversibilité de l'externalisation du pilotage. L'externalisation du pilotage implique en effet une organisation du consortium qui soit adaptée aux différentes tâches à prendre en charge. Un certain degré de coordination interne doit ainsi être assuré afin de maintenir le projet dans les cadres imposés par le dispositif contractuel et ainsi respecter les engagements pris. En complément, une instance de décision a été désignée afin d'arbitrer entre le respect des cadres et l'adaptation aux évènements qui viennent perturber le déroulement du projet. La distribution, interne au consortium, de ces différentes responsabilités assure le maintien d'un certain équilibre entre engagements initiaux et évènements imprévus, ce qui garantit un pilotage pragmatique et efficace.

La contrainte de suivi et de respect des engagements a donc été déléguée dans un premier temps au dispositif sociotechnique contractuel et intégrée ensuite par le consortium dans sa structure. Elle est ainsi prise en compte par deux types différents d'acteurs au sein du collectif qui pilote le projet. Cette appropriation de la contrainte par les acteurs renforce la qualité du management du projet et permet à l'administration communautaire de se reposer sur le collectif, ce qui explique l'efficacité d'un contrôle ponctuel. Cependant l'alourdissement lié à l'externalisation des tâches de coordination et de pilotage d'un projet de recherche communautaire pose aux membres du consortium de recherche des problèmes d'organisation du travail scientifique. Si le temps dédié au pilotage du projet s'accroît, c'est au détriment de celui de la recherche. Aider les chercheurs à se dégager, au moins partiellement, de ces obligations de management, comme le permet par exemple le recours à la sous-traitance, semble être un moyen de garantir la qualité du travail scientifique. Il s'agit de donner les moyens aux chercheurs de maintenir un équilibre entre qualité managériale et qualité scientifique. Piloter un projet de recherche communautaire, c'est donc trouver un modus vivendi entre recherche et règlement, c'est-à-dire entre les sphères scientifique et administrative.

\section{RÉFÉRENCES}

CALLON (M.), 1999. «La sociologie peut-elle enrichir l'analyse économique des externalités? Essai sur la notion de cadrage-débordement ». In: FORAY (D.), MAIRESSE (J.) (Ed.), Innovations et performances. Approches interdisciplinaires, Paris, Éditions de l'EHESS, pp. 399431.

DUPUY (J.-P.), EYMARD-DUVERNAY (F.), FAVEREAU (O.), ORLÉAN (A.), SALAIS (R.), THÉVENOT (L.) (dir), 1989. «L'économie des conventions » (numéro spécial), Revue Économique, 40(2).

FORAY (D.), 2000. L'économie de la connaissance, Paris, La Découverte.

LASCOUMES (P.), LE GALÈS (P.), (dir.), 2004. Gouverner par les instruments, Paris, Presses de Science Po.

LATOUR (B.), 1993a. " "Le groom est en grève. Pour l'amour de Dieu, fermez la porte" ». In: LATOUR (B.) (Ed.), Petites leçons de sociologie des sciences, Paris, La Découverte, pp. 56-76.

LATOUR (B.), 1993b. « Le topofil de Boa Vista - montage photo-philosophique ». In : CONEIN (B.), THÉVENOT (L.), DODIER (N.) (Ed.), Raisons Pratiques. Les objets dans l'action. De la maison au laboratoire, Paris, Éditions de l'EHESS, pp. 187-216.

LATOUR (B.), WOOLGAR (S.), 1988. [1979]. La vie de laboratoire. La production des faits scientifiques, Paris, La Découverte. 
LEIGH STAR (S.), GRIESEMER (J.), 1989. «Institutional Ecology, 'Translation' and Boundary Objects: Amateurs and Professionnals in Berkerley's Museum of Vertebrate Zoology, 1907$1939 »$, Social Studies of Science, 19(3), pp. 387-420.

NORMAN (D.), 1992. Turns Signals are the Facial Expressions of Automobiles, Cambridge, Westview Press.

PARADEISE (C.), 1998. «Pilotage institutionnel et argumentation». In : BORZEIX (A.), BOUVIER (A.), PHARO (P.) (Ed.), Sociologie et cognition, Paris, CNRS Édition, pp. 205-228. 